Proceedings of the 2013 Winter Simulation Conference

R. Pasupathy, S.-H. Kim, A. Tolk, R. Hill, and M. E. Kuhl, eds

\title{
SIMULATION AND OPTIMIZATION OF TEMPORARY ROAD NETWORK IN MASS EARTHMOVING PROJECTS
}

\author{
Chang Liu \\ Ming Lu \\ University of Alberta \\ 116 Street 85 Ave \\ Edmonton, Alberta, CANADA T6G 2W2
}

\author{
Sam Johnson \\ North America Construction Group \\ 11405 - 163 Street \\ Edmonton, Alberta, CANADA T5M 3 Y3
}

\begin{abstract}
Haulage cost typically accounts for around $30 \%$ of the total cost of mass earthmoving projects. The temporary road network is a major factor influencing haulage cost and production efficiency. The simulation of earthmoving operations considering temporary road networks, not only facilitates the site formation design but also leads to realistic, cost-effective construction plans. Utilizing the Floyd-Warshall algorithm and linear programming, this study formulates the temporary road network problem and sheds light on the potential benefits of selecting routes and directions for handling earthmoving jobs. An optimization approach for temporary road networks is further proposed. It reduces the total cost of the project and shortens its duration. Simulation models were used to prove the effectiveness and feasibility of optimization.
\end{abstract}

\section{INTRODUCTION}

Recent research (Kang et al. 2013) has proposed an optimization approach for the layout of haul roads, which are long distance routes between one origin and one destination in mining projects, through automation. Temporary road networks between multiple origins and multiple destinations, however, are relatively short, and are more complicated for mass earthmoving projects. A temporary road network is essentially a site layout problem which determines the hauling routes and directions to move the earth at highest efficiency. Temporary road network designs still mainly rely on experience up to date. Seldom have previous research attempts in earthmoving simulation or earthmoving optimization deliberately considered the temporary road network.

Earthmoving simulation is common and essential in the construction domain. It plays a fundamental role in optimizing the productivity, efficiency and cost of earthmoving operations, by modeling interactive resource cycles and uncertainties in a construction system. Simulation is a powerful tool originally used to obtain the optimum resource allocation by modeling the earthmoving operations in resource-based simulations (Shi and AbouRizk 1994; Hajjar and AbouRizk 1997; Shi and AbouRizk 1998). Genetic Algorithm or other heuristic optimization algorithms are later combined with simulation models to optimize the earthmoving operations (Marzouk and Moselhi 2003; Cheng et al. 2005; Moselhi and Alshibani 2009; Lin et al.2012). With the development of 3D animation in simulation, the 3D motion paths of trucks have been visualized in discrete-event simulation (Kamat and Martinez 2008).

The previous simulation models have largely simplified the hauling routes and directions used in moving the earth. Several studies into earthmoving optimization, however, have focused on optimized routes and directions. Mayer and Stark (1981) raised the issue of minimizing costs through a rational transportation of materials between cut and fill sections. At that time, "mass diagram" was widely used while the linear programming model for cut and fill optimization could not be easily solved due to the low calculation speed of computers (Jayawardane and Harris 1990). Son et al. (2005) successfully used a line- 
ar programming model to determine the minimum haul distance and identify directions to move the earth. Their approach provides a detailed plan describing both material amounts and destinations to haul, which is claimed to make projects more profitable. Xavier de Lima et al. (2012) further developed an innovative approach to optimizing excavation and paving services based on the linear programming model. These optimization approaches are constructive but very likely fall short in addressing uncertainties. In addition, they assume distances between cells to be straight line distances while ignoring the temporary road network in the field. In fact, the temporary road network design fundamentally influences routes and directions to move the earth, and average haul time, especially for mass excavation and site grading projects.

In recent years, research on earthmoving operations have turned to positioning and tracking technologies. Kang et al. (2009) confirmed that in a GIS environment a planner could effectively conduct earthwork planning. To optimize earthmoving operations, Moselhi and Alshibani (2009) obtained the hauling paths based on geographic information systems in their simulation model. Montaser and Moselhi (2012) further established a RFID system to track productivity in near-real-time. Most recently, Kang et al. (2013) developed a GIS-based method to design and optimize haul road layout. The optimization approach depends on linguistic evaluations whose accuracy relies on expert knowledge. Choi and Nieto (2011) utilized Dijkstra's Algorithm and the least-cost path selection to improve the route of trucks in mining sites based on GPS. The least-time routes or least-fuel routes are assigned to trucks according to data, including the existing haul route, road grade, equipment and other information, which is collected in order to conduct an optimized earthwork planning.

Given that previous simulation and optimization approaches have not considered temporary road network design in details, the objective of this paper is to present a method of optimizing earthmoving operations that addresses the above stated limitations and is of use to contractors who have a practical need to improve temporary road network design.

\section{METHODOLOGY}

The proposed methodology is illustrated in Figure 1. In mass earthmoving projects, the field is generally divided into cells, and the cut and fill volume of each cell can be easily obtained. Given a temporary road network, shortest haul time between cells can be obtained by applying the Floyd-Warshall algorithm. The linear programming model further creates the earthmoving plan. In order to achieve a better earthmoving plan, the optimization of temporary road network design is developed through "multi-generation competing" genetic algorithms (MCGA). Simulation models encoded with earthmoving plans are established in order to validate the approach.



Figure 1: Methodology flow chart. 
Chang, Lu, and Johnson

\subsection{Achievement of Earthmoving Plans}

\subsubsection{Floyd-Warshall Algorithm}

An earthmoving plan is the optimal plan for earthmoving operations, allowing the construction mangers to save hauling costs. It provides detailed volumes and directions to move materials. The related field of earthwork optimization offers some theoretical considerations. Son et al.(2005) introduced the concept of "haul effort", which is formulated in (1) in order to optimize the directions for moving the earth.

$$
\bar{D}=\sum_{i=1}^{m} \sum_{j=1}^{n} D_{i j} V_{i j} / \sum_{i=1}^{m} \sum_{j=1}^{n} V_{i j}
$$

Where $\bar{D}$ is the average haul distance, $D_{i j}$ is the distance between the $\mathrm{i}$-th and j-th cell and $V_{i j}$ is the volume to be moved between the $\mathrm{i}$-th and $\mathrm{j}$-th cell. This method uses straight-line distances between cells regardless of the temporary road network. In addition, it does not factor in those factors influencing the haul time, such as grade and rolling resistances. In order to improve the method, the Floyd-Warshall algorithm is introduced to take into account the temporary road network in earthmoving operations.

The Floyd-Warshall algorithm (also known as Floyd's algorithm, Floyd 1962) is a graph analysis algorithm used to determine shortest paths, and it is based on a weighted graph with positive or negative edge weights. Figure 2 gives an example of the Floyd-Warshall algorithm. If a weighted graph (Figure 2a) or edge weight matrix is known, the shortest distance matrix (Figure 2b) can be obtained through the algorithm.

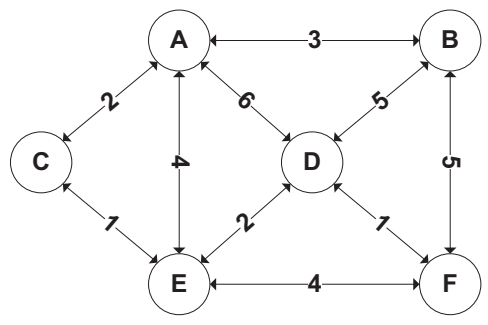

Fig 2a: Weighted graph.

\begin{tabular}{l|llllll} 
& $\mathrm{A}$ & $\mathrm{B}$ & $\mathrm{C}$ & $\mathrm{D}$ & $\mathrm{E}$ & $\mathrm{F}$ \\
\hline $\mathrm{A}$ & - & 3 & 2 & 5 & 3 & 6 \\
$\mathrm{~B}$ & 3 & - & 5 & 5 & 6 & 5 \\
$\mathrm{C}$ & 2 & 5 & - & 3 & 1 & 4 \\
$\mathrm{D}$ & 5 & 5 & 3 & - & 2 & 1 \\
$\mathrm{E}$ & 3 & 6 & 1 & 2 & - & 3 \\
$\mathrm{~F}$ & 6 & 5 & 4 & 1 & 3 & -
\end{tabular}

Fig 2b: Shortest distance matrix.

Figure 2: Floyd-Warshall algorithm example.

The Floyd-Warshall algorithm can provide the shortest distances or times between cells for a temporary road network. The proposed approach defines the weight of each edge to be the shortest haul time between cells. The weights can be calculated following the equation which is given in (2).

$$
w_{i j}=d_{i j} / v_{i j}
$$

Where $d_{i j}$ is the distance between the $\mathrm{i}$-th and $\mathrm{j}$-th cell and $v_{i j}$ is the average haul speed between the $\mathrm{i}$ th and $\mathrm{j}$-th cell. It is noteworthy that the meaning of output matrix can be changed if the definition of weight is different. The edge weight calculation can be further developed and determined by total resistance, distance, average hauling speed and better expert knowledge.

\subsubsection{Linear Programming Model}

The Floyd-Warshall algorithm provides insight into the temporary road network in earthmoving optimizations. The linear programming model, which is given in (3), is established to generate an earthmoving plan with the minimized average haul time. The established model can be programmed and accomplished by mathematical software designed for solving linear programming problems. The outputs of linear programming model can be grouped together to represent the optimized earthmoving plan.

$$
\min \sum_{i=1}^{n} \sum_{j=1}^{m} s_{i j} V_{i j} / \sum_{i=1}^{n} \sum_{j=1}^{m} V_{i j}
$$


Chang, Lu, and Johnson

$$
\begin{array}{ll}
\text { s.t. } & \sum_{j=1}^{m} V_{i j}=V_{i-\text { cut }}, 1 \leq i \leq n \\
& \sum_{i=1}^{n} V_{i j}=V_{j-\text { fill }}, 1 \leq j \leq m \\
& V_{i j} \geq 0, s_{i j} \geq 0,1 \leq i \leq n, 1 \leq j \leq m
\end{array}
$$

Where $s_{i j}$ is the shortest haul time between the i-th and j-th cells obtained from the Floyd's algorithm, $V_{i j}$ is the volume to be moved between the i-th and j-th cell, $V_{i \text {-cut }}$ is the total cut volume of the i-th cell and $V_{j-\text {-fil }}$ is the total fill volume of the $\mathrm{j}$-th cell.

\subsection{Optimization of Temporary Haul Road Network}

Nowadays, the earthmoving plan can be optimized thanks to the calculation speed of mathematical software. Temporary road networks, however, can hardly be optimized because it mainly relies on experience and there is no specific design guideline.

For a detailed design, the main temporary road is generally designed to connect massive cut areas with massive fill areas, and meanwhile some other finger roads are designed to be connected with the main road. The empirical design method is concluded on the basis that (1) the cost of temporary road network is equal to or less than the reduction in hauling costs resulting from evaluating various temporary road network design scenarios; and (2) the project can be finished within scheduled time, which means the cost in connection with building and operating the temporary road network is also within the scope.

This assumption is practically simple and effective, but it cannot be generalized to a new project easily, especially for construction managers with limited experience or over complicated site grading or mass earthmoving jobs. In such situations, choosing different temporary road network design may lead to considerable cost saving. In the present research, the optimization approach is proposed, aimed to find a controllable and analytical method for construction managers, planners and decision makers to improve overall project cost-efficiency by designing better temporary road networks. The optimization approach, which is essentially a cost-benefit analysis, is established based on conceptual models of road network.

\subsubsection{Objective Function}

The objective of building the temporary road network is to accelerate the project progress while keeping a limited budget. The objective function is aimed to search for the optimized temporary road network resulting in the lowest cost while also meeting the requirement of project duration. In the objective function, the project duration is defined as the average haul time which is given in (4) and obtained from the linear programming model. The total cost function given in (5) consists of the costs for establishing a temporary road network, the maintenance costs and the costs of equipment and crew. It is noted that the number of trucks should be determined for specific cases rather than ideal truck excavator combinations. Recent research (Morley et al. 2013) stated that there is no predefined rules for optimal combination.

$$
\begin{gathered}
\bar{T}=\sum_{i=1}^{n} \sum_{j=1}^{m} s_{i j} V_{i j} / \sum_{i=1}^{n} \sum_{j=1}^{m} V_{i j} \\
C_{t}=C_{c}+C_{m}+C_{e} \cdot \bar{T} \cdot \frac{\sum V_{i j}}{n \cdot c}
\end{gathered}
$$

Where $\bar{T}$ is the average haul time obtained from the linear programming model, $s_{i j}$ is the shortest haul time between the $\mathrm{i}$-th and $\mathrm{j}$-th cell obtained from the Floyd-Warshall algorithm, $V_{i j}$ is the volume to be moved between the i-th and j-th cell, $C_{t}$ is the total cost related to the temporary road network, $C_{c}$ is the construction and removal costs of the temporary road network, $C_{m}$ is the maintenance, risk and other costs, $C_{e}$ is the hourly or daily total costs of equipment and crew, $n$ is the number of trucks, and $c$ is the capacity of a truck. The function of maintenance, risk and other costs given in (6) is assumed to vary depending on the scale of temporary road network and it is costly for the trucks to haul on the rough ground, due to the higher risk and shorter life cycle of tires. 


$$
C_{m}=C_{m-t}+p \cdot\left(C_{m-r}-C_{m-t}\right)
$$

Where $C_{m-t}$ is the maintenance, risk and other costs if trucks always haul on temporary road network, $C_{m-r}$ is the maintenance, risk and other costs if trucks always haul on rough ground and $p$ is the scale of temporary road network in percentage depending on its total length.

\subsubsection{Temporary Haul Road Network Model}

In the optimization approach, temporary road network design is deemed as $0-1$ knapsack problems. The conceptual model is established and each dash line between centroids of cells means a decision whether to build the temporary road. The temporary road network can also be represented in the format of number series such as $[0,1,0, \ldots, 0,0,1]$, which will be used in the genetic algorithm to represent potential solution. " 0 " means remaining rough ground between $\mathrm{i}$-th centroid and $\mathrm{j}$-th centroid. " 1 " means the temporary road is available between $\mathrm{i}$-th centroid and $\mathrm{j}$-th centroid. Figure 3 illustrates the derivation of temporary road network model. For each cell, the centroid is simplified to be the geometric center of cell and the potential road network can be observed in Figure 3b.
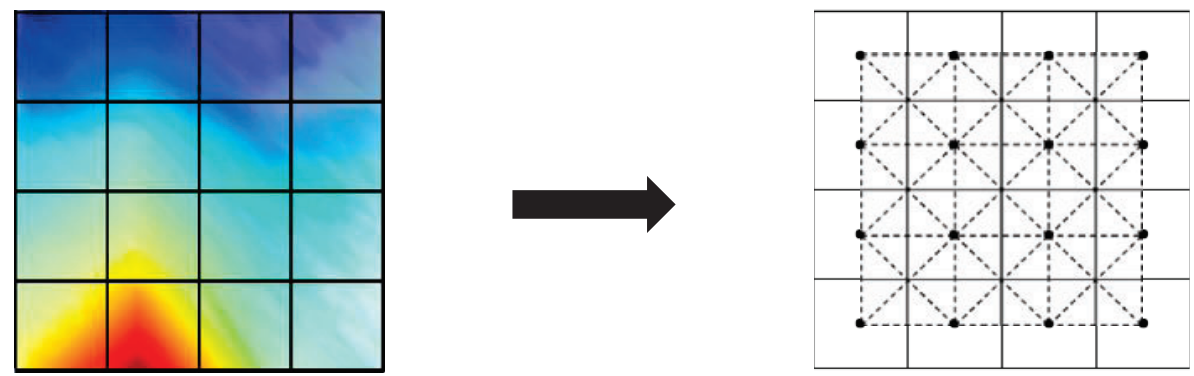

Figure 3a: Cells overlaid on the field with cut and fill areas. Figure 3b: Network model overlaid on cells. Figure 3: 0-1 Model for temporary road network design.

\subsubsection{Optimization Based on Genetic Algorithm}

The optimization is established through heuristic searching algorithms. The multi-generation compete genetic algorithm (MCGA, Deng et al. 2007) is chosen to search the optimized temporary road network. The optimization approach was programmed and accomplished in MATLAB (MATLAB 2012). Genetic algorithms has the limitation that it converges towards a local optimum instead of the global optimum of the problem. MCGA, whose parameters are listed in Table 1, can address this limitation to some extent and it inherits the advantages including faster searching speed and easier to achieve the global optimum.

Table 1: Parameters of multi-generation compete genetic algorithm.

\begin{aligned} \hline Variable & Description \\ \hline $\mathrm{N} &$ Size of chromosomes depending on the temporary road network size; \\ MP & Size of multi-generation; \\ NIND & Number of individuals; \\ GGAP & Generation gap; \\ MAXGEN & $\begin{array}{l}\text { Termination criteria which means the length of time during which minimum } \\ \text { value remains the same; }\end{array}\end{aligned}$

The flow chart which is illustrated in Figure 4 indicates the proposed optimization approach. After setting the input data including the cut and fill volume of each cell, the parameters of genetic algorithm and empirical parameters of the objective function, the algorithm starts to search for the optimized temporary road network. When the termination criteria is reached, the optimal solution is obtained. The optimal solution should meet the requirement of average haul time, which is $T$ in the Figure 4, with the lowest to- 
tal costs in the objective function. After multiple runs of the optimization approach, from random start points, the optimized temporary road network can be derived from limited alternatives.

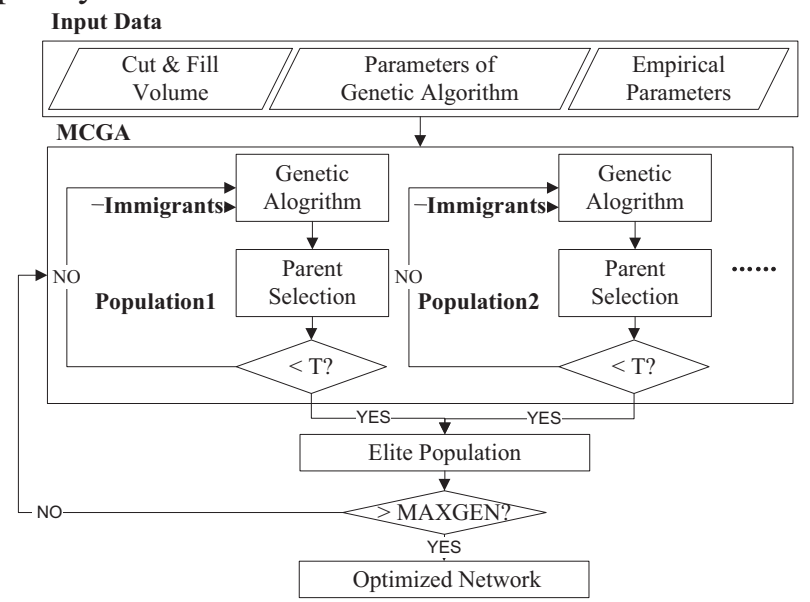

Figure 4: Flow chart of MCGA optimization approach.

\section{A CASE STUDY IMPLEMENTING PROPOSED APPROACHES}

\subsection{Overview of Earthmoving Project}

To illustrate and verify the proposed approaches, a case study based on a rough grading project was chosen. The rough grading project is the preliminary work package of a camp site construction in Fort McMurray, AB. The field which is around 120 hectares is divided into 48 cells whose spacing is $150 \mathrm{~m}$ by $150 \mathrm{~m}$. The project has around $335,000 \mathrm{~m}^{3}$ of earthwork.

Input data of the proposed method are cut and fill volumes, speed conditions of trucks, empirical data and temporary road network model. Designed cut and fill areas are illustrated in Figure 5 whose volumes are measured in cubic meters of undisturbed soil given in Table 2. The speeds of trucks are listed in Table 3 and the mode speeds under different operation conditions are retrieved from contractors' past experience. The imported materials are assumed to be counted into volume of the cell located at the entrance. The temporary road illustrated in Figure $6 \mathrm{a}$ is designed based on past experience which can be converted into the model of temporary road network illustrated in Figure 6b. Figure 6 demonstrates the establishment of the empirical network model which is further embellished by applying the proposed method.

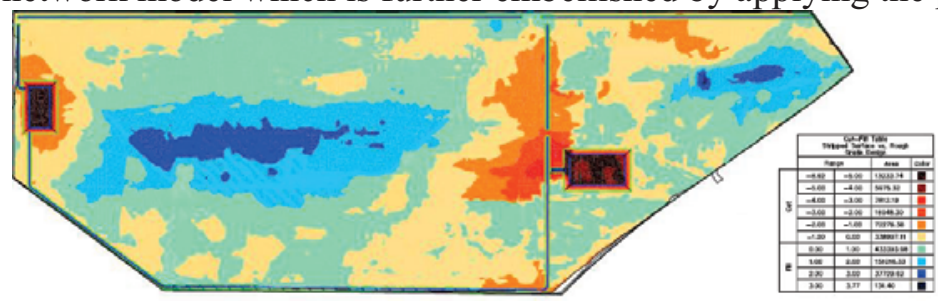

Figure 5: Designed cut and fill areas of rough grading design.

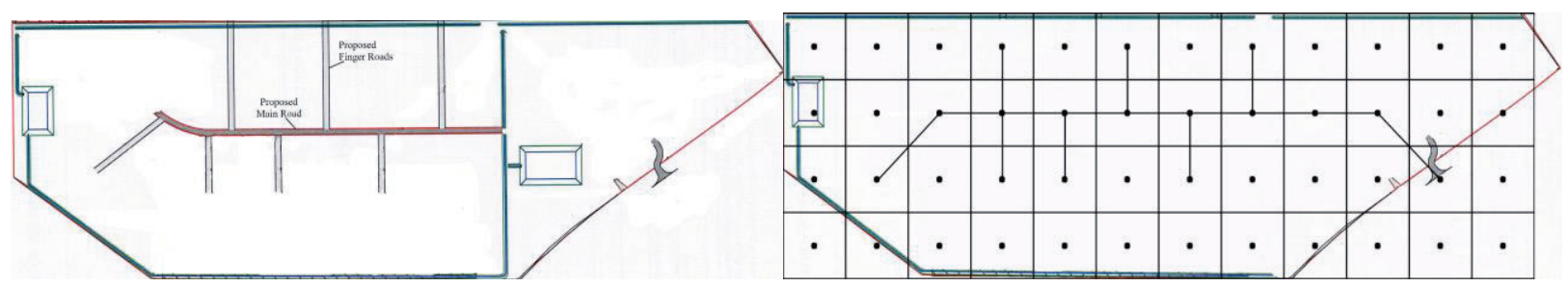

Figure 6a: Empirical temporary road network. Figure 6b: Network model to be used in proposed approach.

Figure 6: Establishment of the model for empirical temporary road network. 
Chang, Lu, and Johnson

Table 2: Cut and fill volumes of each cell $\left(100 \mathrm{~m}^{3}\right)$.

\begin{tabular}{|c|c|c|c|c|c|c|c|c|c|c|c|c|}
\hline Cell No. & 37 & 38 & 39 & 40 & 41 & 42 & 43 & 44 & 45 & 46 & 47 & 48 \\
\hline Volume & 150 & 37 & -37 & -90 & -90 & -80 & 10 & 112 & 23 & 220 & 69 & -112 \\
\hline Cell No. & 25 & 26 & 27 & 28 & 29 & 30 & 31 & 32 & 33 & 34 & 35 & 36 \\
\hline Volume & 626 & -225 & -338 & -360 & -230 & -222 & -81 & 248 & 99 & 22 & -143 & -79 \\
\hline Cell No. & 13 & 14 & 15 & 16 & 17 & 18 & 19 & 20 & 21 & 22 & 23 & 24 \\
\hline Volume & 37 & -225 & -281 & -230 & -243 & -142 & 124 & 344 & 725 & 285 & 25 & 0 \\
\hline Cell No. & 1 & 2 & 3 & 4 & 5 & 6 & 7 & 8 & 9 & 10 & 11 & 12 \\
\hline Volume & 0 & 14 & -23 & -12 & -90 & 59 & 99 & 27 & -23 & 1 & 0 & 0 \\
\hline
\end{tabular}

Table 3: The average haul speed of trucks.

\begin{tabular}{rcl}
\hline Operation Condition & Speed Type & Mode $(\mathrm{km} / \mathrm{h})$ \\
\hline Rough ground & Haul & 17 \\
Rough ground & Return & 24 \\
Temporary road & Haul & 31 \\
Temporary road & Return & 42 \\
\hline
\end{tabular}

\subsection{Achievement of Earthmoving Plans}

Earthmoving plans are determined for two scenarios. One scenario considers temporary road network in earthmoving operations and the other scenario does not consider road network. It is assumed that centroids are simplified to be the geometric center of each cell. The weight of each road section is calculated by using Equation 2 based on the average haul speed and distances between centroids which are $150 \mathrm{~m}$ in this case. Through the proposed approach, the optimal solutions of earthmoving plans with minimized average haul time are achieved.

When considering the temporary road network, average haul time is $1.346 \mathrm{~min}$. If the road network is neglected, average haul time is $1.637 \mathrm{~min}$. The difference is about $18 \%$ and therefore temporary road networks should not be negligible in earthmoving simulation or optimization analysis.

\subsection{Optimization of Temporary Road Network}

The optimized temporary road network is obtained following the proposed optimization approach. In this case, cut and fill volumes are given and the input parameters set for genetic algorithms are determined after trials ( $\mathrm{NIND}=80 ; \mathrm{N}=146 ; \mathrm{MP}=20 ; \mathrm{GGAP}=0.9$; $\mathrm{MAXGEN}=20$ ). The parameters of the objective function are determined by empirical data (Construction and removal costs of the temporary road is $\$ 16,000 / 100 \mathrm{~m}$; Maintenance, risk and other costs for temporary road is $\$ 400 /$ day and for rough ground is $\$ 1200 /$ day; Daily cost of equipment and crew is $\$ 3,200 /$ day; 8 trucks; 8 working hours/day). The project is assumed to be handled by 50 -ton trucks whose capacity is $30 \mathrm{~m}^{3}$. The working efficiency is not considered in this case. The limit of the average haul time is set in the model. After multiple runs, several optimal solutions can be obtained. Considering the constructability of the temporary road network, the optimized temporary road network model is identified as demonstrated in Figure 7.

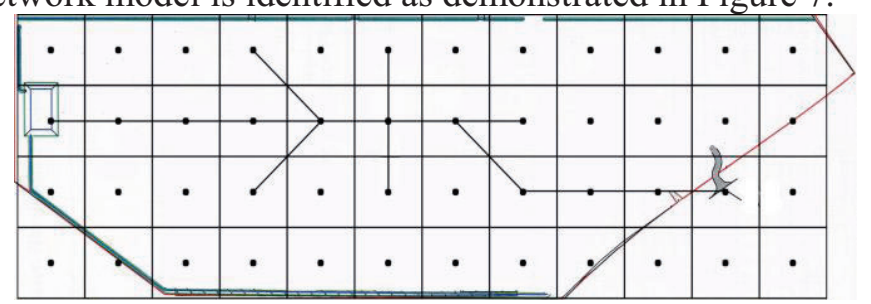

Figure 7: Temporary road network model based on optimized solutions. 
The optimized temporary road network model, which is the output of proposed method, seems to be more complicated than the empirical model. The similarity is that the massive cut areas is also connected with the massive fill area in the optimal solution. The temporary road network can be further designed in detail based on this optimized model. Given the optimized temporary road network in the project, average haul time reduces to $1.096 \mathrm{~min}$.

\subsection{Simulation with Earthmoving Plans}

The simulation models are established to encode optimized earthmoving plans. The general purpose template of Simphony, a discrete-event modeling environment, is used to establish the simulation models as shown in Figure 8. The main element in Simphony is "Task" which represents the activity with the duration distribution. The "Capture" and "Preempt" represent the resource requirements for "Task". In this case, haul time and return time are coded into "Task" elements based on the earthmoving plans.

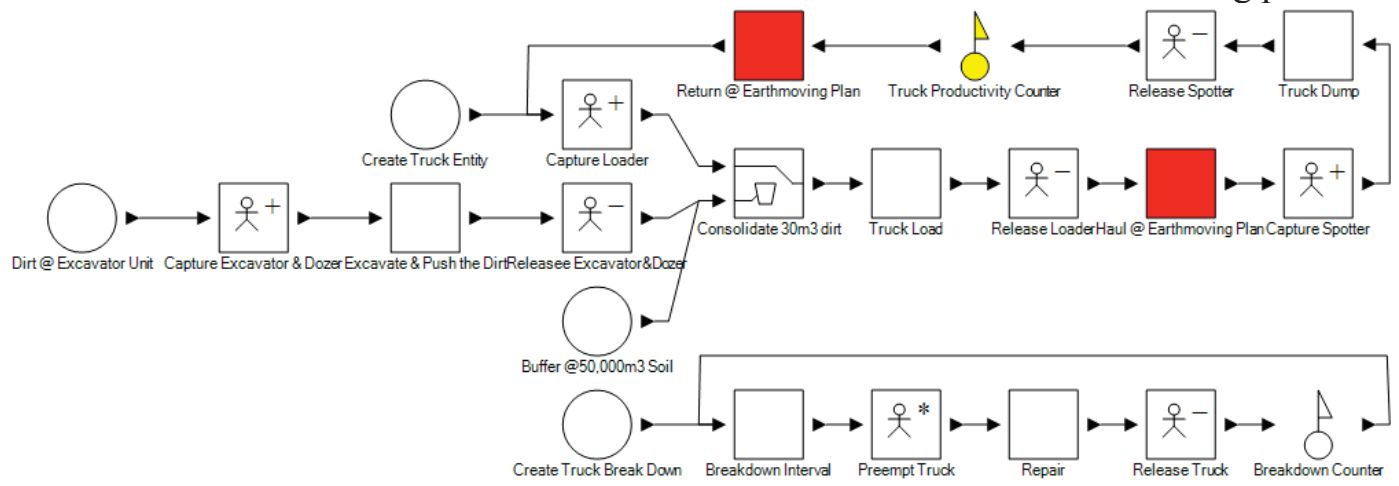

Figure 8: Basic model in Simphony encoded with earthmoving plan.

The duration distribution, capacity and resource involved in the tasks are listed in the Table 4, whereas duration distributions are retrieved from the empirical data. They are used as the input data for simulation. It is noteworthy that haul times and return times are obtained from the proposed approach. The simulation is assumed to be executed by 8 trucks, 4 excavators, 4 dozers, 4 loaders and 4 spotters. The excavators and dozers start to excavate and push $10 \mathrm{~m}^{3}$ soil to the loading location each time. After excavating $50,000 \mathrm{~m}^{3}$ soil, the loaders are allowed to load an empty truck with $30 \mathrm{~m}^{3}$ soil. The trucks haul soil to the fill area and dump the soil under the inspection of a spotter. The truck returns to the loading location to continue the earth moving cycle. Trucks break down occasionally and repair time is required to fix the truck. The duration distributions of activities listed in the Table 4 are used in the model.

Table 4: The duration, capacity and resource of tasks.

\begin{tabular}{lllll}
\hline No. & Task name & Resource & Duration (min) & Capacity $\left(\mathrm{m}^{3}\right)$ \\
\hline 1 & Excavate \& & Excavator(1), & Triangular $(1.6,4,3)$ & 10 \\
2 & Push the dirt & Dozer(1) & Triangular $(1.5,2.1,1.8)$ & 30 \\
3 & Haul Load & Loader(1), Truck(1) & Obtained from proposed approach & 30 \\
4 & Truck Dump & $\operatorname{Truck}(1)$ & Truck(1), Spotter(1) & Triangular $(1.8,2.5,2)$ \\
5 & Return & $\operatorname{Truck}(1)$ & Obtained from proposed approach & 30 \\
6 & Breakdown Interval & $\operatorname{Truck}(1)$ & Triangular $(300,480,360)$ & N/A \\
7 & Repair & $\operatorname{Truck}(1)$ & Triangular $(60,150,80)$ & N/A \\
\hline
\end{tabular}




\section{VALIDATION OF OPTIMIZATION APPROACH}

If earthmoving simulations encoded with earthmoving plans pass verification and validation, the entire optimization approach can be deemed useful. The optimized temporary road network performs better than the empirical network by comparing the average haul time. In order to validate the approach, three simulation models encoded with corresponding earthmoving plan are developed as follows:

1. Model encoded with earthmoving plan without considering temporary road network.

2. Model encoded with earthmoving plan considering empirical temporary road network.

3. Model encoded with earthmoving plan considering optimized temporary road network.

The outputs of simulation models including mean duration and productivity are listed in Table 5 . The "average haul time" is the output from linear programming model. The cost of the temporary road network is obtained based on the objective function which is given in (5).

Table 5: Comparison between three models.

\begin{tabular}{ccccc}
\hline $\begin{array}{c}\text { Model } \\
\text { No. }\end{array}$ & $\begin{array}{c}\text { Mean } \\
\text { Duration (h) }\end{array}$ & $\begin{array}{c}\text { Mean } \\
\text { Productivity }\left(\mathrm{m}^{3} / \mathrm{h}\right)\end{array}$ & $\begin{array}{c}\text { Average Haul } \\
\text { Time }(\mathrm{min})\end{array}$ & $\begin{array}{c}\text { Costs of Road } \\
\text { Network }(\$)\end{array}$ \\
\hline 1 & 474.89 & 706.50 & 1.637 & 22,000 \\
2 & 423.68 & 790.67 & 1.346 & 285,000 \\
3 & 391.64 & 855.98 & 1.096 & 273,000 \\
\hline
\end{tabular}

The mean duration to finish earthwork is about 10\% shorter when comparing Model 2 (423.68h) against Model 1 (474.89h). Influenced by the production rate of excavators, the difference between durations become smaller but still significant. Therefore the temporary road network should not be negligible in earthmoving simulation models. The mean duration to finish earthwork is about 7\% shorter when comparing Model 3 (391.64h) against Model 2 (423.68h). And the cost of optimized temporary road network is about $5 \%$ lower than the empirical temporary road network. The optimized solution shows its advantages in accelerating the project with a lower cost based on simulation.

\section{CONCLUSION}

The proposed approach was successfully applied to an site grading project for achieving the earthmoving plan and optimizing the temporary road network. The simulation models in Simphony validate the necessity of consideration of temporary road network in earthmoving simulations and the effectiveness to the obtain the optimized temporary road network. The planners or decision-makers can take advantage of the earthmoving plan to lower the earthwork costs through the proposed approach. The engineers with limited experience on temporary road network design can achieve a convincing solution by simply utilizing empirical costs. In addition, the approach is also suitable for quantitatively comparing several layouts of the temporary road network.

As the optimization approach cannot be detached from the empirical cost data and the conceptual model of temporary road network, it is foreseen that there is a need to improve the reliability of empirical cost data to achieve better optimized solutions. The space of cells varies depending on conditions of test cases which should be further studied. In addition, the efficiency of the optimization algorithm can be improved, which depends on complexity of the problem definition. The further improvement of the proposed approach would be worthy of research to help construction managers rely on a controllable and analytical method rather than experience for critical decision making in earthmoving planning.

\section{REFERENCES}

Deng, L., Shi, F., and Liang, T. (2007) "Multi-Generation Compete Genetic Algorithms and Its Application in Vehicle Routing Problem. " International Conference on Transportation Engineering 2007: pp. 3184-3189. 
de Lima, R., Júnior, E., de Athayde Prata, B., and Weissmann, J. (2012). "Distribution of Materials in Road Earthmoving and Paving: A Mathematical Programming Approach." J. Constr. Eng. Manage, 10.1061/(ASCE)CO.1943-7862.0000666 (Dec. 14, 2012).

Floyd, Robert W. (June 1962). "Algorithm 97: Shortest Path". Communications of the ACM 5 (6): 345.

Hajjar, D., and AbouRizk, S. (1997). "AP2-Earth: A Simulation Based System for the Estimating and Planning of Earth Moving Operations." Winter Simulation Conference, 1103-1110.

Kamat, V. R., and Martinez, J. C. "Generic representation of 3D motion paths in dynamic animations of simulated construction processes." Autom.Constr., 17(22), 188-200.

Kang, S., and Seo, J. (2013). "GIS Method for Haul Road Layout Planning in Large Earthmoving Projects: Framework and Analysis." J.Constr.Eng.Manage., 139(2), 236-246.

Kang, S., Seo, J., and Baik, K. (2009) "3D-GIS Based Earthwork Planning System for Productivity Improvement. " Construction Research Congress 2009: pp. 151-160.

Lin, C. , Hsie, M., Hsiao, W., Wu, H. , and Cheng, T.(2012). "Optimizing the Schedule of Dispatching Earthmoving Trucks through Genetic Algorithms and Simulation." J.Perform.Constr.Facil., 203-211.

Marzouk, M., and Moselhi, O. (2003). "Object-oriented Simulation Model for Earthmoving Operations." Journal of Construction Engineering \& Management, 129(2), 173.

Marzouk, M., Moselhi, O., Moselhi, O., and Alshibani, A. (2009). "Object-oriented Simulation Model for Earthmoving Operations." Journal of Construction Engineering and Management-Asce, 129; 135(2; 10), 173; 948-954.

Montaser, A. and Moselhi, O. (2012) "RFID+ for Tracking Earthmoving Operations. " Construction Research Congress 2012: pp. 1011-1020.

Morley, D., Lu, M. and Joseph, T. (2013). "In search of the Ideal Truck-Excavator Combination. " International Symposium on Automation and Robotics in Construction 2013.

Shi, J., and AbouRizk, S. M. (1994). "A resource based simulation approach with application in earthmoving/strip mining." Winter Simulation Conference, 1124.

Shi, J., and AbouRizk, S. S. (1998). "An Automated Modeling System for Simulating Earthmoving Operations." Computer-Aided Civil \& Infrastructure Engineering, 13(2), 121.

Smith, S. D., Son, J., Mattila, K. G., and Myers, D. S. (2005). "Earthmoving productivity estimation using linear regression techniques." Journal of Construction Engineering and Management-Asce, 125; $131(3 ; 3), 133 ; 302-309$.

Son, J., Mattila, K. G., and Myers, D. S. (2005). "Determination of haul distance and direction in mass excavation." Journal of Construction Engineering and Management-Asce, 131(3), 302-309.

Tao-ming Cheng, Chung-wei Feng, and Yan-liang Chen. "A hybrid mechanism for optimizing construction simulation models." Autom.Constr., 14 85-98.

\section{AUTHOR BIOGRAPHIES}

CHANG LIU is a MSc student at the Hole School of Construction Engineering in the Department of Civil and Environmental Engineering at the University of Alberta. His research interests are optimization of earthmoving operations and construction simulation. His email address is chang4@ualberta.ca.

MING LU is an Associate Professor in the Department of Civil \& Environmental Engineering at the University of Alberta. His research interests are construction surveying and automation; operations simulation and scheduling in construction. His email address is mlu6@ualberta.ca.

SAM JOHNSON is a Project Manager with North American Construction Group and graduate of the Construction Engineering MEng program at the University of Alberta. His primary research interests lie in continuous improvement of construction operations through practical application of operations simulations and project controls systems. His email address is sjohnson@nacg.ca. 